\title{
FACTEURS FAVORABLES A LA TRANSMISSION DE LA FILAIRE DE LABORATOIRE DIPETALONEMA DESSETAE *
}

\author{
par G. Petit, O. Bain et H. Spitalier-Kaveh \\ Laboratoire de Zoologie (Vers) associé au C.N.R.S., \\ Muséum d'Histoire naturelle, 43, rue Cuvier, F 75231 Paris Cedex 05.
}

Nous avons précédemment proposé la Filaire Dipetalonema dessetae Bain, 1973, comme modèle pour les travaux sur les filarioses expérimentales (Bain, 1973). Cette Filaire a, en effet, pour avantages :

a) un hôte naturel, Proechimys guyanensis (E. Geoffroy), qui s'élève sans aucune difficulté au laboratoire ;

b) des vecteurs, Aedes aegypti ou A. polynesiensis, qui sont également faciles à élever ;

c) une microfilarémie périodique avec un pic diurne situé entre $12 \mathrm{~h}$ et $14 \mathrm{~h}$;

d) une sensibilité aux filaricides actuels comparable à celle des Filaires humaines (Gayral et Pommiès, 1976).

Cette Filaire, transmissible par Moustiques et non par Acariens, ayant une microfilarémie périodique, se rapproche donc plus des espèces pathogènes pour l'Homme que les modèles sur Rongeurs qui étaient disponibles précédemment (Litomosoides carinii, D. viteae).

L'entretien de cette souche, effectué au laboratoire depuis cinq ans et par certains collègues auxquels nous l'avons communiquée, a pu être amélioré au niveau du vecteur en tenant compte de trois facteurs:

1) Souche du vecteur: le vecteur qui donne les meilleurs résultats est une souche africaine d'Aedes aegypti, souche Gkep (Ghana, Kpedome), entretenue à la London School of Tropical Medicine et donnée par P. Mac Greevy.

2) Repas sanguins: nous avions indiqué (Bain, 1973) que les stades infestants sont plus robustes si les Moustiques prennent des repas de sang sur Rongeur sain pendant le développement larvaire de la Filaire. L'influence de ces repas sanguins supplémentaires est, en fait, beaucoup plus considérable : avec trois repas, le taux d'infestation des Moustiques est considérablement amélioré, le nombre moyen de stades infestants par femelle est décuplé et les stades larvaires attardés sont beaucoup plus rares (tableau $I$ ). Santé.

* Ces recherches ont pu être faites grâce à une subvention de l'Organisation Mondiale de la Reçu le 10 avril 1977. 
Tableau I. - Effets des repas sanguins sur le taux d'infestation d'A. aegypti Gkep.

- Dans l'expérience 1, les moustiques gorgés sur Proechimys infesté n'ont pas été triés et les résultats sont basés sur l'ensemble des moustiques $q$ récoltés dans la cage à la fin du développement larvaire; dans l'expérience 2, les moustiques gorgés sur Proechimys ont été triés.

(Légendes: Exp.: $\mathrm{n}^{\circ}$ d'expérience; Mf: nombre de microfilaires dans $10 \mu \mathrm{l}$ de sang du Proechimys; (ㅇ): nombre d'A. aegypti $q$ disséqués; Inf: nombre moyen de stades infestants par moustique femelle; \% S.a.: stades larvaires attardés exprimés en pourcentage du nombre total de larves).

\begin{tabular}{|c|c|c|c|c|}
\hline Exp. & Mf & pas de & repas sur & Cobaye \\
\hline & & Inf. & $\%$ Sa. & \\
\hline 1 & 586 & 0,49 & $\overline{77}$ & $(55$ ㅇ) \\
\hline 2 & 811 & 1,3 & 72 & (76 \$) \\
\hline
\end{tabular}

\begin{tabular}{ccc}
3 & repas & sur Cobaye \\
\hline Inf. & $\%$ S.a. & \\
3,32 & $\overline{-}$ & $(78$ \%) \\
10,9 & 7,3 & $(78$ \%) \\
\hline
\end{tabular}

3) Température: un inconvénient de cette Filaire était la lenteur du développement larvaire chez le Moustique : 26 jours à $23^{\circ} \mathrm{C}$ chez A. aegypti Gkep. En élevant la température à $26^{\circ} \mathrm{C}$, la durée du développement s'abaisse à 18 jours, le taux d'infestation des Moustiques restant aussi bon (tableau II).

Tableau II. - Effet de la température sur l'infestation d'A. aegypti Gkep.

- Dans les expériences à $26^{\circ} \mathrm{C}$, le gorgement et la traversée de la paroi digestive - environ les 17 premières heures - se sont déroulés à $23^{\circ} \mathrm{C}$.

(Microfilarémie du Proechimys: 1014 microfilaires/10 $\mu \mathrm{l}$. Durée : temps du développement jusquà la forme infestante, exprimée en jours).

\begin{tabular}{cccc} 
Exp. & \multicolumn{3}{c}{$23^{\circ} \mathrm{C}$} \\
\hline & Durée & Inf. & \\
1 & 26 & 7 & $(150$ \%) \\
2 & 26 & 6,1 & $(85 \%)$ \\
\hline
\end{tabular}

\begin{tabular}{cll}
\multicolumn{3}{c}{$26{ }^{\circ} \mathrm{C}$} \\
\hline Durée & Inf. & \\
18 & 6,36 & $(146 \%)$ \\
18 & 5,24 & $(92 \%)$ \\
\hline
\end{tabular}

A condition de tenir compte de ces trois facteurs, nous croyons donc pouvoir confirmer l'excellente qualité de Dipetalonema dessetae comme modèle pour les Filarioses expérimentales.

\section{Bibliographie}

BaIN (O.), 1973. - Une nouvelle Filaire de Rongeur sud-américain, Dipetalonema dessetae n. sp. (Nematoda, Filarioidea). Bull. Mus., 3e sér., 116, Zool., 90, 309-316.

Bain (O.), 1973. - Cycle d'une Filaire de Rongeur passant par Moustique. C.R. Acad. Sci. Paris, 276, sér. D, 2689-2690.

Gayral (P.) et Pommiès (M.), 1976. - Résultats préliminaires sur l'utilisation d'une nouvelle Filaire de Rongeur, Dipetalonema dessetae (Bain, 1973) dans l'évaluation des antifilariens. C.R. Acad. Sci., 283, sér. D, 861-864. 